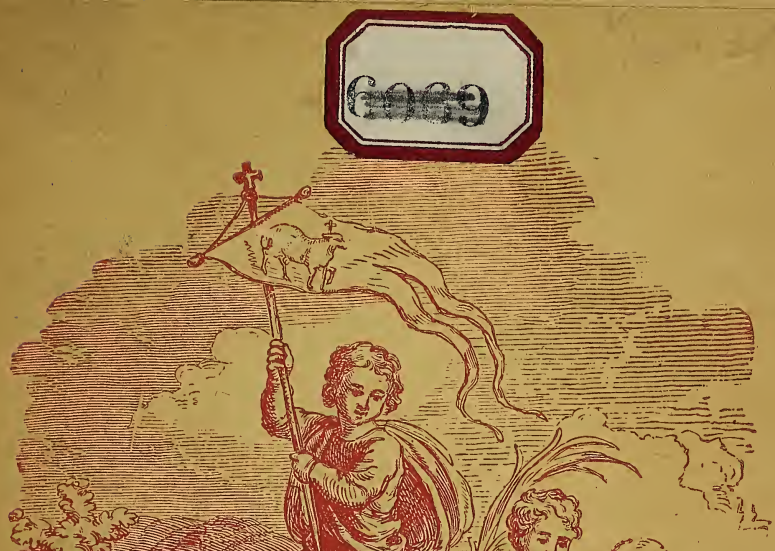
3.

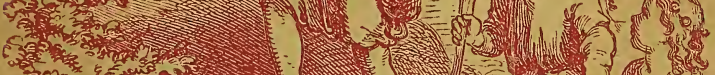

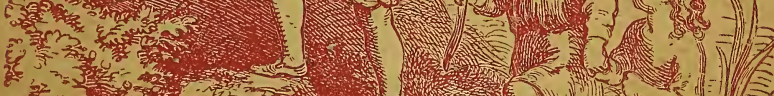

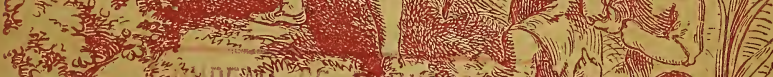
6. We tris ans.:sion -

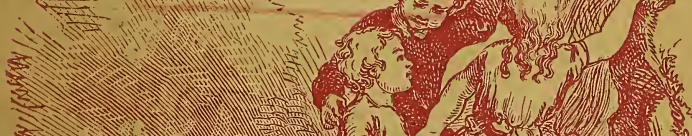
ș

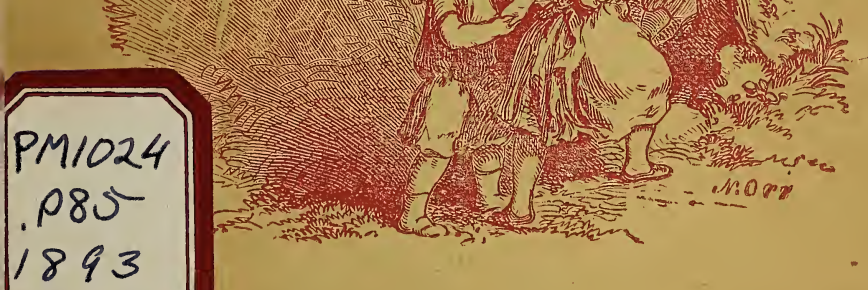





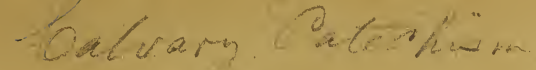

\title{
CALIARY
}

1893

\section{WIWIGAWANGAPI KIN,}

\section{WOKIKSUYE ANPETI KIN KOYA.}

Mrs. D. C. WESTON, Owa, REV. JOSEPH W. COOK,

$$
\text { QA }
$$

Mr. CHARLES S. COOK,

\author{
IESKA KAGAPI.
}

"Nakun taku ota eciciyapi kta bduha, tka hena e qe nahanrcin yuha oyalcihipi $x$ ni."-Sт. JoHN xvi:12.

NIOBRARA DEANERY OF SOUTH DAKOTA.

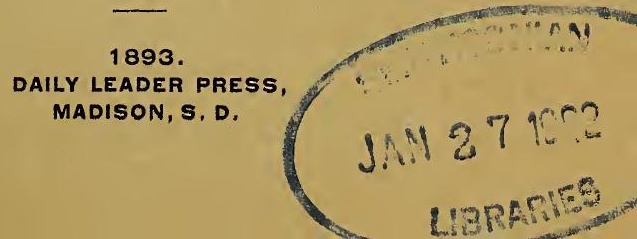




\section{PM

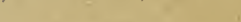 893}

\section{Galvart! WiWicaWangani Kin.}

\section{WOONSPE I.}

1. Tuwa maka kin de kaga he? Wakantanka.

2. Anpetu tukte wanji ed okirpa he? Anpetu ixakowin.

3. Anpetu kin he toked eciyapi he? Anpetu Okirpapi.

4. Anpetu tokaheya kin ed Wakantanka taku kaga he? Iyoyanpa.

5. Iyoyanpa kin he Wakantanka toked caxton he? Anpetu.

6. Oiyokpaze cin ix toked caxton he? Hanhepi.

7. Anpetu icinonpa kin ed taku kaga he? Okotonyan kin.

8. Okotonyan eyapi kin he taku kapi he? Marpiyato kin. 


\section{WOONSPE $1 \mathrm{I}$.}

1. Anpetu iciyamni kin ed Wakantanka taku kaga he? Maka kin mini akna.

2. Anpetu icitopa kin ed Wakantanka taku kaga he? Anpetu-wi, hanhepi-wi, wicarpi ko.

3. Wanji tuktee iyoyanpa tanka eciyapi he? Anpetuwi kin.

4. Unma aoptetu kin ix toked caxton he? Hanhepi-wi.

5. Anpetu icizaptan kin ed Wakantanka taku kaga he? Hogan qa warupakoza.

6. Anpetu icixakpe kin ed taku kaga he? Woteca kin.

7. Anpetu icixakpe kin ed nakun taku kaga he? Wicaxa.

8. Wicaxa tokaheya kin he Wakantanka toked caxton he? Adam.

9. Adam, winyan tokaheya kin toked caxton he? Eve.

10. Adam taku etanhan kagapi he? Maka bdu kin.

11. Eve ix taku etanhan kagapi he? Adam cutuhu wanji etanhan.

\section{WOONSPE III.}

1. Wakantanka Adam qa Eve taku owicaniya he? Wiconi taniya kin.

2. Hetanhan takupi he? Wicanagi niun kin hecapi.

3. Wakantanka, Adam qa Eve tukted ewicaknaka he? Maga wan mahed.

4. Maga kin he toked eciyapi he? Eden. 


\section{4}

5. Wakantanka taku econwicaxi he? Maga kin he awanyagwicaxi.

6. Adam qa Eve waxtepi he?. Han, toka qehan.

7. Toked on iyohakab Wakantanka toiye eced econpi xni he? Waskuyeca wan yutexniwicaxi qon he yutapi.

8. Tuwa takaheya he yuta he? Eve.

9. Yutin kta e tuwa Eve iyutanyan he? Satan.

10. Tokae he yutin kta cin he? On ksapin kta kecin.

11. Tuwa waskuyeca kin he etanhan Eve yudkiya he? Adam.

\section{WOONSPE IV.}

1. Wana yutapi qehan toked econpi he? Naicirmanpi.

2. Tokae naicirmanpi he? Wakantanka itkokipapi kte cin kokipapi.

3. Toked iyopewicaya he? Maga kin etanhan karab iyewicaya.

4. Tokae ake maga kin ed kdapi kta okihipi xni he? Maga tiyopa kin natakapi.

5. Tuwa maga tiyopa kin awanyakapi he? Cherubim.

6. Hena takupi he? Oknikde.

7. Nakun taku on awanyakapi he? Miwakan ide wan on.

8. Nakun toked iyopewicaya he? Tapi kta Wakantanka kewicakiya.

9. He taku e Wakantanka iwahowicaya he? Wanikiya wan.

10. Adam qa Eve cinca yamnipi kin toked ewicakiyapi he? Cain, Abel qa Seth. 


\section{5 \\ WOONSPE.V.}

1. Wicaxa ikduota ayapi qonhan ocib xica qaix waxtepi he? Ocib xicapi.

2. Wakantanka tuked ecawicakieon kta keya he? Ihangwicayin kta keya.

3. Wakantanka taku e makata uya he? Minitan wan.

4. Tuwepi e minitan kin etanhan niwicayapi he? Noah tiyoknaka ob.

5. Wakantanka Noah taku e karxi he? Wata wan.

6. He taku wata he? Witawata tanka.

7. Taku Noah wata kin ed owicaknaka he? Wamanica ocaje owasin etanhanhan.

8. Anpetu tona maka kin amagaju he? Anpetu wikcemna-topa.

9. Anpetu tona mini kin maka akand waxagya wanka he? Opawinge sanb wikcemna zaptan.

10. Wata kin he wan toked eciyapi e ed azi he? Ararat.

\section{WOONSPE VI.}

1. Anpetu wikcemna topa qehan Noah taku e tankad iyayeya he? Kangi wan.

2. Nakun taku e tankaù iyayeya he? Tidwakiyenawan.

3. Tidwakiyena kin he kangi kdi xni qon ix iyeced econ he? Hiya, kdi.

4. Anpetu tona. Noah ape qa hehand ake tidwakiyena kin iyayeya he? Anpetu xakowin.

5. Taku kakdi he? Can-wikdi ape wan. 
6. Noah tankad hiyu qehan taku kaga he? Waknawoxnapi wan.

7. He akand Noah taku e wayurtata he? Worurnarye woxnapi wan.

8. Wakantanka marpiya xapa kin ekta taku e eknakin kta keya he? Wikmunke wan.

9. He taku on wapetokeca kta he? Icimana minitan uyin kte xni e on.

10. Tohand wikmunke kin he wanunyakapi ecee he? Bdoketu ehand magaju akisni eca.

11. He toked cajeunyatapi he? Wikmunke.

WOONSPE VII.

1. Minitan xni itokab wicaxa tohanhanyan nipi s'a he? Ota waniyetu opawinge napciwang aktonktonyan.

2. Wakantanka tohind wicaxa tonipi kin yuptecena he? Minitan kin iyohakab.

3. He ehan iapi tonakeca on iapi he? Wanjina.

4. Wakantanka tohind iapi toktokeca on iewicakiya he? Babel kaga yukanpi icunhan.

5. Tokae hecon he? Nakicironpi kte xni e.

6. Hehand taku akipapi he? Maka owancaya obdecahan hinkdapi.

7. Babel eyapi kin he taku he? Tipestona wankandtuya.

8. Tipi kin he tohan wankandtuya kta oyate kin cinpi he? Marpiya kin ihunniyan.

\section{WOONSPE VIII.}

1. Wakantanka taoyate ehannapi kin toked ewicakiyapi he? Hebrews, Jews qa Israelites. 


\section{7}

2. Tuwa itancankiyapi qa woope wicaqu he? Moses.

3. Hunkakewicaye cin hena tuwepi he? Amram qa Jochebed.

4. Juda oyate kin ed tuwa tokaheya woxnakaga itancan he? Aaron, Moses ciyeku kin.

5. Tukte makoce owanyagwaxte kae ekta Moses Juda oyate kin awicai he? Canaan makoce kin.

6. Waniyetu tonakeca qonhan Egypt etanhan Canaan ed ihunnipi he? Waniyetu wikcemna topa.

7. Moses te cehan Judah oyate kin tuwa itancankiyapi he? Joshua qa Wayasupi kin.

8. Wayasupi tona wicayuhapi he? Akezaptan.

9. Ohanketa taku cinpi he? Wicaxayatapi wanji.

\section{WOONSPE IX.}

1. Israel oyate kin tuwa tokaheya wicaxayatapi yuhapi he? Saul, Kish cinhintku kin.

2. Wicaxayatapi inonpa qon he tuwe he? David, Jesse cinhintku kin.

3. Wicaxayatapi iyamni qon be tuwe he? Solomon, David Cinhintku kin.'

4. Solomon taku wan kaga he? Tipi-wakan tanka tokaheya kin.

5. Tukted kaga he? Jerusalem ed.

6. Taku on kagapi he? Inyan ska.

7. Taku on owanyagwaxte kagapi he? Mazaskazi, mazaska qa inyan oteririka. 
8. Wakantanka tokedked oyate waonspewicakiya ecee he? Wowihanbde qa wowanyake on.

9. Nakun toked? Waayatapi kin eciyatanhan.

10. Waayatapi kin taku tokonpis'a he? Taku tokata hiyohi kte cin hena ayatapi ece e.

11. Wakantanka ehaker tuwa uxi he? Iye Cinhintku Jesus Christ.

\section{WOONSPE X.}

1. Wanikiya unkitawapi kin he tuwe he? Wakantank? Cinhintku hecena kin hee.

2. Tukted he unyawapi he? Wowapi Wakan kin ed.

3. Toked eciyapi he? Jesus Christ.

4. Tohind Jesus tonpi he? Waniyetu 1800 sam iyeya.

5. Anpetu wan ed tonpi qon he wokiksuye kin tukte e he? December 25.

6. He anpetu kin toked cajeunyatapi he? Christmas.

7. Otonwe wan ed tonpi qon he toked eciyapi he? Bethlehem.

8. Bethlehem eciyapi kin he tuktetu he? Judea makoce kin ed.

9. Otonwe kin he ed tukted tonpi he? Waniyanpi tipi wan ed.

10. Tukted eonpapi he? Waniyanpi okna wotapi wan ed.

\section{WOONSPE XI.}

1. Jesus Christ hunku kin tuwe he? Mary, Witanxnaun kin. 
2. Tukte wicowazi hae Mary Witanxna-un kin etanhan he? David Wicaxayatapi wicowazi tawa kin.

3. Tuwepi e tokaheya Jesus tonpi kin owicakiyakapi he? Tarcaxunka awanwicayakapi qeya.

4. Hehan taku tokonpi he? Tarcaxunka optaye kin awanwicakdakapi.

5. Tuwa he owicakiyaka he?' Oknikde wakan wan.

6. Tuwa Oknikde wakan kin de uxi he? Wakantanka.

7. Taku e aokdutewicaya he? Iyoyanpa wiyakpa wan.

8. Unkan toked canteyuzapi he? Wikopapi.

9. Oknikde wakan kin toked eya he? Wikopapi xni po.

10. Taku wicakahi keya he? Wotanin waxte.

11. Wotanin waxte kin he nakun tuwa yuhapi kta keya he? Maka sitomniyan oyate hiyeye cin owasin.

\section{WOONSPE XII.}

1. Tarcaxunka awanyakapi kin tokiya Jesus wanyag ipi he? Bethlehem ekta.

2. Nakun tuwa Jesus wanyag hipi he? Wicaxa ksapapi qeya.

3. Tokiyatanhan hena upi he? Wiyohiyanpatanhan.

4. Taku e wanke cin ed awicai he? Wicarpi wan.

5. Toked he ya he? Wicitokab.

6. Wicaxa ksapapi kin Jesus taku qupi he?' Mazaskazi, pejuta waxtemna qa canxin.

7. Wicaxa ksapapi kin kikdapi qonhan Joseph ed tuwa ikdutanin he? Oknikde wakan wan.

8. He Joseph taku econxi he? Inajin qa iyayexi.

9. He tokiya yexi he? Egypt ekta. 
10. Tuwa yuha yexi he? Jesus hokxicopa kin he.

11. Nakun tuwa? Hanku kin.

WOONSPE XIII.

1. Joseph tokiya Jesus ayexipi he? Egypt ekta.

2. He tokae Jesus Egypt makoce kin ekta ayexipi he? Niyanpi kta e.

3. He ehan tuwa wicaxayatapi he? Herod.

4. He Jesus taku ecakicon kta cin he? Kte kta cin.

5. Jesus taku ki kta Herod iye ikopa he? Iye tokiconze kin.

6. Herod te cehan Joseph toked toran he? Ake icicawin Juda makoce kin ekta kdi.

7. Tuwa Herod tohe qon okna yanka he? Archelaus.

8. Tukte otonwe kae ed Jesus ounyan he? Nazareth.

9. Tohanyan hed un he? Wana wicaxa hehanyan.

10. Nazareth otonwe kin he tukte etu he? Galilee makoce kin ed.

11. Nazareth otonwe kin tanka qaix ciscina he? Ciscina.

WOONSPE XIV.

1. Wicaxa wan Jesus itokab hi qon he tuwe he? John Baptisma wicaqu qon hee.

2. He taku iwahokonwicakiya he? Woiyopeiciye.

3. John taku wokoyakeya he? Woteca wan camel eciyapi e hin kin heca.

4. Taku woyuteya he? Psipsicana qa turmaga-canhanpi.

5. John wahokonwicakiya eca nakun taku econ he? Baptisma wicaqu ece. 


\section{1}

6, Jesus John ekta i he? Han.

7. Taku on Jesus hed ya he? Baptisma qupi kta on.

8. Tokae hecon he? Waonspeunkiyapi kta e on.

9. He taku e tidwakiyena iyeced Jesus ed hiyahan he? Wakantanka Taniya Wakan kin.

10. Marpiya eciyatanhan wicaho wan toked eya he? Micinkxi waxtewakidake cin he dee ce.

\section{WOONSPE XV.}

1. Jesus waniyetu tonakeca ehand waonspewicakiya he? Waniyetu wikcemna yamni ecetu ehand.

2. Tohanyan waonspewicakiya he? Waniyetu yamni.

3. Waonspewicakiya eca nakun taku econ ece e he? Wapetogya wicor'an econ ece e.

4. Hehan taku he? Woyuxinyaye wicor'an kin.

5. Tona ee he? Wicaxa tapi kex kiniwicaya ece e.

6. Qa nakun taku he? Leper ececapi kin wicayuska ece e.

7. Ixtagongapi kin hena ix taku ecawicakicon he? Tonwewicaya ece e.

8. Huxtepi kin ix taku ecawicakicon he? Maniwicaya ece e.

9. Ia okihi xni kin ix toked ecawicakicon he? Iewicakiya ece e.

10, Tate qa taja kin toie eced econpi he? Han.

\section{WOONSPE XVI.}

1. Jesus tuwa kici unpi kta e wicakico he? Wahoxiye wakan akenonpapi kin. 
2. Hena taku wanyakapi kta cin he? Woyuxinyaya wicoran Tawa kin.

3. Taku nar'onpi kta cin he? Wuwaxte wicoie Tawa kin.

4. Hena wanyakapi qa nar'onpi kinhan takupi kta he? Waayatanin Tawa kin heepi kta.

5. Taku on hena waeconwicaxi he? Wotanin-waxte kin oyakapi kta e.

6. Hena taku icaryapi kta he? Okodakiciye wakan kin.

7. Taku econpi kta e on wowaxake wicaqu he? Wayazanpi kin asniwicayapi kta.

8. Wanji tukte wamnayanpi kin heca he? Saint Matthew.

9. Saint Peter qa Saint Anàrew takupi he? Hokuwa wicaxa kin hecapi.

10. Nakun tuwa hokuwa wicaxa kin hecapi he? Saint James qa Saint John.

\section{WOONSPE XVII.}

1. Tokae Jesus ouncage unkitawapi kin icu he? Niunkiyapi kta e..

2. Jesus eyapi kin he toked kapi he? Wanikiya.

3. Jesus ktepi qon he ehan Juda oyate kin ed tuwa itancankiyapi he? Pontius Pilate.

4. He ehan tuwa woxna kaga itancan he? Caiaphas.

5. Tuwa he Jesus knayan wicaqu he? Judas Iscariot.

6. He tuwe he? Wahoxiye wakanpi kin wanji ee.

7. Waonspewicakiyapi kin Itancan unkitawapi irpeya iyayapi xni he? Hiya, irpeya iyayapi.

8. Tukte maga kae ed Jesus nina iyokixid un he? Gethsemane. 


\section{3}

9. Tokae tin kta rea he? Woartani unkicicajujupi kta e.

10. He toked econ okihi he? Unkiyepi eekiya Iye kakixyapi kin heon.

\section{WOONSPE XVIII.}

1. Judas, Jesus knayan wicaqu kin on taku qupi he? Mazaska wikcemna yamni.

2. Taku on knayan wicaqu he? Woiiputake on.

3. He icunhan maga wan Jesus ed un qon he toked eciyapi he? Gethsemane.

4 Tuwa Jesus partapi qa akiyakdapi he? Wicaxa oxpaye wan.

5. Wicaxa kin hena tuwa uwicaxi he? Woxnakaga itancanpi kin.

6. Hena tuwa Jesus kaipi he? Pilate.

7. Pilate, Jesus taku erakicon he? Kaxkagnaka.

8. Nakun taku ecakicon he? Can wan ed icipawer okatanpi kta e wicaqu.

9. Nakun taku Jesus ecakiconpi he? Wapepeka texdagkiyapi.

10. Qa nakun taku ecakiconpi he? Atagoxaxapi.

\section{WOONSPE XIX.}

1. Wanikiya canicipawega tawa kin tuwa aya he? Iye rca katehanyanna se kdoi.

2. Tuwa okiya he? Simon, Cyrene etanhan kin.

3. Jesus toked nagi kin ed kakija he? Nina iyokixica.

4. Ohanketa taku ecakiconpi he? Icipawer okatanpi. 
5. He toked econpi he? Canicipawega wan akand okatanpi.

6. Tohanyan canicipawaga kin ed okatan wanka he? Wihiyayena oape xakpe.

7. Jesus taku yatkin kta e qupi he? Mini-xkumna.

8. Anpetu tukte wanji ed canicipawega ed okatanpi he? Anpetu izaptan kin.

9. Tukte ed kakija he? Calvary Paha kin akand.

10. Jesus kici canicipawega ed owicakatanpi qon hena tuwepi he? Wamanonpis'a nonb.

\section{WOONSPE XX.}

1. Wamanonpi s'a kin unma rcin iyopeiciya he? Han, unma hecon.

2. Unkan woartani kicicajujupi he? Han.

3. Canicipawega kin ed tuwa ehake yankapi he? Mary yamnipi kin.

4. Nakun tuwa he? Saint John.

5. Jesus toked eciya he? Nihun wankdaka yo.

6. He tuwa ke ca heya he? Mary Witanxna-un kin.

7. Hehand John toked econxi he? Awanyagxi.

8. Jesus canicipawega ed okatanpi qon hena taku ecawicakicon he? Wocekiye ewicakiciciya.

9. Toked eya he? Ate wicakicicajuju ye.

10. Tuwa Jesus tancan kin ra he? Joseph Arimathea makoce etanhan kin.

11. Rapi kin ed tuwa okiya he? Nicodemus. 


\section{5 \\ WOONSPE XXI.}

1. Jesus tancan kin Joseph taku iyapemni he? Miniruha ska.

2. Tukted eonpa he? Okna wicarapi teca rca wan iye tawa e ed.

3. Okna wicarapi kin he taku etanhan-najin kagapi he? Inyan.

4. He taku e Joseph icapan elkde he? Inyan tanka wan.

5. Inyan kin he akand taku eknaka he? Wiyapuspe.

6. Rapi kin ed tuwa awanyagwicakiyapi he? Akicita waawanyakapi oxpaye wan.

7. Jesus nagi kin tokiya i he? Paradise ekta.

8. Tancan kin tohanyan wicarpi kin ed wanka he? Anpetu izaptan qa Anpetu-wakan hehanyan.

9. Anpetu iciyamni kin ed taku toketu he? Jesus wiconte kin etanhan inajin.

10. Anpetu-wakan oko ed anpetu kin wanji tukte ed kini he? Anpetu tokaheya kin.

\section{WOONSPE XXII.}

1. Anpetu-wakan oko kin ed anpetu tokaheya kin he toked eciyapi he? Anpetu Wakan, qaix Sunday.

2. Jesus anpetu wan ed kini qon he toked eciyapi he? Easter-anpetu.

3. Easter eyapi kin taku kapi he? Inajinpi.

4. Anpetu-wi kin tohand yanka ehand Christ kini he? Hinhanna rcin. 
5. Easter hinhanna rce cin ed tuwa marpiya eciyatan han hi he? Oknikde wakàn wan.

6. Oknikde wakan kin taku tokon he? Rapi kin etanhan inyan kin yutokand iyeya.

7. Hehand oknikde wakan kin taku tokon he? Inyan kin akand iyotanka.

8. Tuwa tokaheya Jesus wanyaka he? Mary Magdalene.

9. Iyohakab tuwa wanyakapi he? Waonspewicakiya akewanjipi kin.

10. Judas toked ohranyan he? Icirte.

\section{WOONSPE XXIII.}

1. Itancan kini kin iyohakab hunkawanjidkiciyapi tona witaya wanyakapi he? Opawinge zaptan.

2. Kini kin he eciyatanhan taku unkonspepi he? Tye Wakantanka Cinhintku kin hee kin he.

3. Jesus taku iwahounyanpi he? Unlix eya unkinipi kte cin he.

4. Tohan kinhan unkinipi kta he? A npetu ehake kin ed.

5. Jesus kini kin iyohakab anpetu tona maka akand un he? Anpetu wikcemna-tob.

6. Anpetu kin hena ed taku econ le? Waonspewicakiyapi Tawa kin waonspewickiya.

7. Taku econwicaxi te? Wotanin waxte kin oyagwicaxi.

8. Nakun taku econpi kta he? Oyate owasin Baptisma wicaqupi kta. 
9. Tuwa Caje eciyatanhan? Ateyapi kin, qa Cinhintku kin, qa Woniya Wakan kin.

10. Jesus taku uwicakiyin kta keya he? Woniya Wakan kin.

\section{WOONSPE XXIV.}

1. Anpetu wikcemna topa qehan taku toketu he? Jesus marpiya kin ekta iyaya.

2. Tuktetanhan Jesus wankand iyaya he? Olive Paha kin etanhan.

3. Paha kin he tukte etu he? Jerusalem ikiyena.

4. Tuwa Jesus kici unpi he? Waonspewicakiye cin.

5. Wankand iyaye cin wanyakapi he? Han.

6. Jesus wana irpeya wicakiyakni kte cehan taku tokon he? Wicayawaxte.

7. He taku e isinyan iyaye ca on wanyakapi xni he? Marpiya xapa wan.

8. Jesus tokiya wankand iy aya he? Wakantanka taonspeyapata.

9. Tohind Woniya Wakan kin waonspewicakiye cin ed wicai he? Christ wankand iyaye ciqon iyohakab anpetu wikcemna qehan.

10. Wokiksuye anpetu wanji tukte etu he? Pentecost anpetu kin.

\section{WOONSPE XXV.}

1. Dehand Jesus tokiya un he? Wakantanka taonspeyapatanhan.

2. Taku tokon yanka he? Waceunkiciciyapi. 


\section{8}

3. He on etanhan toked eciyapi he? Wawiciya unkitawapi.

4. Jesus tohand ake $\mathrm{u}$ kta he? Maka owihanke kinhan.

5. Toked u kta he? Kikde ciqon he ake iyeced.

6. He toked kapi he? Marpiya kin eknayan u kta kapi.

7. Tuwa kici upi kta he? Oknikde wakanpi kin.

8. Tuwa mnawicayin kta he? Wicaxa maka oknaka kin owasin.

9. Taku on hecon kta he? Wicayasu kta e.

10. Toked wicayasu kta he? Tor'anpi kin iyacinyan.

\section{WOONSPE XXVI.}

1. Tona waxtepi kin Christ tukted ewicaknakin kta he? Iye taonspeyapatanhan.

2. Tona xicapi kin ix tukted ewicaknakin kta he? Iye tacatkayapatanhan.

3. Waxtepi kin hena taku ewicakiyin kta he? "Ate niyawaxtepi kin, u po."

4. Xicapi kin hena taku ewicakiyin kta he? "Niyaxicapi kin, Mitantanhan iyaya po."

5. Anpetu kin he on toked piunkiciyapi kta he? Christ wacinunyanpi kte cin on.

6. Nakun taku econqonpi kta he? Wakantanka Tawoahope kin hena ahounpapi kta.

7. Woahope tokaheya qa tanka kin he tukte e he? Wakantanka waxtedapi kte cin he e.

8. Toked waxteundapi kta he? Uncantepi kin ataya on.

9. Woahope icinonpa kin he tukte e he? Unkikiyenati kin tanyan canteunkiyuzapi kta. 
10. Unkikiyena-ti kin toked canteunkiyuzapi kta he? Unkiyepi iyeced,

\section{WOONSPE XXVIJ.}

1. Wowapi Wakan kin toked econqonpi kta keya he? Woartani unyuhapi kin okdagunxipi.

2. Nakun wicaxa owasin taku econwicaxi he? Iyopeiciyapi qa Baptisma wicaqupi kta.

3. Taku on Baptisma unqupi he? Mini on.

4. Tuwa Caje kin on? Ateyapi kin, qa Cinhintku kin, qa Woniya Wakan kin.

5. Baptisma eyapi kin he taku kapi he? Wowahokiciye.

6. Wowahokiciye eyapi kin he taku kapi he? Anokatanhan taku ecakiciconpi kta keyapi.

7. Baptisma nicupi eca ed taku ecanonpi kta kehapi he? Wicoran xica irpeunyanpi kta.

8. Taku wicayadapi kta kehapi he? Christian wowicada kin.

9. Taku e ahoyapapi kta kehapi he? Wakantanka Tawacin wakan qa woahope Tawa kin.

10. Tonhanyan hena ecanonpi kta kehapi he? "Tohan- yan wani kin hehanyan."

\section{WOONSPE XXVIII.}

1. Baptisma ed taku nicagapi he? Christ ematanhan.

2. Qa nakun taku? Wakantanka cinca.

3. Nakun taku? Marpiya wokiconze kin tawawayin kta. 
4. Baptisma eciyatanhan wowaxte iyotan kin he tukte e he? Woartani etanhan wicayuskapi kin.

5. Wakiconzapi inonpa kin he tukte e he? Itancan Rtayetu Wotapi Tawa kin hee.

6. Tokae Itancanunyanpi kin he kaga he? Iye te cin he kiksuyeunyanpi qa unnagipi kin wicayuwax'akin kta e.

7. Itancan Rtayetu Wotapi Tawa akantanhan kin he taku he? Aguyapi qa minixa.

8. Qa imahetanhan kin ix taku he? Christ Tancan qa We kin.

9. Tona he icupi kin taku econpi kta kewicakiyapi he? Iyopeiciyapi kta, Jesus wacinyanpi kta, qa wicaxa owasin cantewicakiyapi kta.

10- Itancan Rtayetu Wotapi Tawa kin nakun toked eciyapi he? Eucharist.

\section{WOONSPE XXIX.}

1. Okodakiciye wakan tromaka kin ed Anpetu-waken tokaheya kin toked eciyapi he? Advent Anpetu-wakan.

2. Advent eyapi hin he toked kapi he? U kte cin.

3. Advent ed he tuwe e u kte cin iwaunktapi he? Christ.

4. Advent eyapi kin he tohantu he? Christmas itokab anpetu-wakan topa.

5. Advent hihunni kin iecana rcin wokiksuye-anpetu wan toked caxtonpi he? Saint Andrew taanpetu kin.

6. Saint Andrew taanpetu kin tohantu he? November 30th.

7. Saint Andrew eciyapi kin he tuwe he? Wahoxiye wakan kin heca.

8. He tuwa cinhintku he? Jonas Galilee etanhan kin. 


\section{1 \\ WOONSPE XXX.}

1. Tuwa tokaheya Messiah kin iyeya he? Saint Andrew.

2. He tuwa e Christ ed ai he? lye ciyeku Simon.

3. Jesus tak ewicakiya he? "Miyakna u po."

4. Saint Andrew Martyr heca he? Han.

5. Christian Martyr eyapi kin he taku kapi he? Tuwa Christ on ta eca heciyapi.

6. Saint Andrew toked ktepi he? Canicipawega wan ed okatanpi.

7. Tukted heconpi he? Petraea, Achaia makoce kin ed.

8. Akand icipawer okatanpi qon he iyececa wanji wanunyakapi eca toked cajeunyatapi he? Saint Andrew tacanicipawega kin.

9. Wokakije kin he tokedya itkokipa he? Wiyuxkinye rcin.

\section{WOONSPE XXXI.}

1. Saint Thomas taanpetu kin tohantu he? December 21 st.

2. Saint Thomas eciyapi kin he tuwe he? Galilee ed hokuwa wicaxapi kin heca.

3. Christ he taku kaga he? Wohoxiye wakan.

4. Saint Thomas taku cetunkda he? Christ kini kin.

5. Jesus he toked wicadaya he? Nape ed mazaokatanpi otanin kin hena kipazo.

6. Nakun taku kipazo he? Cuwi ocape kin.

7. Saint Thomas tak eciya he? "Itancan mitawa qa mita-Wakantanka kin."

8. Saint Thomas tokiya eta he? India ed.

9. Wowiyuxkin-anpetu iyokihe kin tukte e he? Christ tonpi anpetu kin.

10. He tohantu he? December 25th. 


\section{2}

\section{WOONSPE XXXII.}

1. December 25th toked cajeunyatapi he? Christmas anpetu.

2. Tukte ekta Christ tonpi kin he owapi he? Rome ekta; ecin hed wicayawapi wowapi kin knakapi.

3. Wicayawapi wowapi kin de tohonwind karwicaxipi he? Saint Luke ii:2. Cyrenius Syria makoce ed itancan qon he ehan.

4. Otokahe ekta Christ wicadapi kin toked Christmas ahopapi he? Tipi-wakan yuhapi kin rantexa qa taku hecekceca on kducopis'a.

5. Nakun taku econpi he? Wowiyuxkin odowan ahiyayapi qa Itancan Rtayetu Wotapi Tawa Woecon kin he econpi ece.

6. Xiceca tokae anpetu kin de waxtedapi kta iyececa he? Anpetu kin he ed Christ hokxicopa icage cin heon.

\section{WOONSPE XXXIII.}

1. Saint Stephen taanpetu kin tohantu he? December 26 th.

2. Saint Stephen eciyapi kin he tuwe he? Christian Martyr tokaheya kin hee.

3. Toked ta he? Inyan on katapi.

4. Tuwa ktepi he? Juda oyate kin.

5. Tuwa ed inajin qa ktepi kta e iyowinyan he? Saul, Tarsus etanhan kin.

6. Iyohakab Saul taku he? Christian heca.

7. Saint John taanpetu kin tohantu he? December 27th.

8. Saint John eciyapi kin he tuwe he? Zebedee qa Salome cinhintkupi.

\section{WOONSPE XXXIV.}

1. Saint John taku he? Wahoxiye wakan kin heca. 
2. John cincu qon he toked eciyapi he? James.

3. Christ James qa John akta rcin toked cax wicaton he? Boanerges.

4. He taku kapi he? "Wakinyan cincapi."

5. Wowapi Wakan kin ed John nakun toked eciyapi he? "Waonspekiyapi wan Jesus iyotan kidake ciqon."

6. Wicaxayatapi xica toked Saint John ecakicon he? Wikdi piryahan wan ed oiyorpeya.

7. Wikdi pirya he cin ed ta he? Hiya, ecaca itoketu xni.

8. Toked ta he? Ikceya ta.

9. Waniyetu tonakeca qehan ta he? Opawinge.

\section{WOONSPE XXXV.}

1. Ikcewicaxa rca ekna Wahoxiye wakan un qon he tuwe he? Paul, Tarsus etanhan kin.

2. Tokaheya toked eciyapi he? Saul, Tarsus etanhan kiv.

3. Woohoda tukte wanji kae etanhan he? Juda oyate tawoohodapi kin.

4. Obe tukte wanji? Pharisee.

5. Toked waonspekiyapi he? He ehan wicoksape yuhapi kin oknayan.

6. Wokage warji tukte onspekiyapi he? Wakeya kagapi kin.

7. Saul tona Christian hecapi kin toked wicakuwa he? Xicaya.

8. Tukted tokaheya xicaya wicakuwa he? Jerusalem ed.

9. Hehand tukted i he? Damascus ekta.

10. Tokae heciya i he? Christian tona hed unpi kin hena wicakaxkin kta e.

\section{WOONSPE XXXVI.}

I. Saul, Damascus etkiya ya icunban taku akipa he? 


\section{4}

Marpiya kin eciyatanhan iyoyanpa wan on ixtagonga hin. kda.

2. Wicaho wan taku eciya he? "Saul, tokae xicaya mayakuwa he?"

3. Saul toked ayupta he? "Itancan, nituwe he?"

4. Ix toked ayuptapi he? "Jesus he miye, xicaya yakuwa kin."

5. Hehand toketu he? Damascus ekta yus aipi.

6. Wawanyake xni qon tuwa akisniyan he? Ananias; "Wikcemna xakowinpi kin" wanji ee.

7. Ananias nakun taku ecakicon he? Baptisma qu.

8. De iyohakab Saul toked eciyapi he? Paul.

9. Wowapi Wakan kin ed wowapi kin tuktena Paul owa he? Wowapi wicakicagapi aketob.

10. Paul toked ta he? Rome ed, pa kaksapi.

\section{WOONSPE XXXVII.}

1. February 2 d, ed wokiksuye anpetu kin he toked eciyapi he? Christ tipi-wakan ed aipi kin.

2. February 24 th ed wokiksuye anpetu kin he toked eciyapi he? Saint Matthias taanpetu kin.

3. Judas icikte qonhan wahoxiye wakanpi kin taku econpi he? Wanji tokeca harnigapi.

4. Tokae? Judas tohe kin oxtan ekdepi kta e.

5. Toked karnigapi he? Woohiye irpekiyapi.

6. Woohiye kin tuwa ahinrpaya he? Matthias.

7. Matthias eciyapi kin he tuwe he? Waonspewicakiya wikcemna xakowinpi kin wanji ee.

8. Taku on he waayatanin kin heca he? Christ kini kin qa marpiyata kikde cin.

9. Matthias toked ta he? Nakun ix eya Martyr heca. 


\section{5 \\ WOONSPE XXXVIII.}

1. Wetu kin ed akiraniciyapi wan ahopapi kin he toked eciyapi he? Lent.

2. Lent woakiraniciye kin he anpetu tonakeca he? Wikcemna topa.

3. Tuwa anpetu wikcemna tob akiraniciya he? Jesus Christ.

4. Tukted akiraniciya he? Hewoskand.

5. Lent ed anpetu tokaheya kin he toked eciyapi he? Ash-Wednesday.

6. Easter Anpetu kin tohantu he? Juda oyate ta Woacakxin Wotapi kin iyohakab anpetu wanji.

7. Woacakxin Wotapi kin tohand hi ece kin taku on sdodyapi he? Hanhepi-wi kin eciyatanhan.

8. Wi iyohi wi tanin ece kin he ocib tokeca aya eca taku toketu he? Ix eya iyeced Easter anpetu kin anpetu tokeca wanji ehand hihunni ece.

9. Lint ed anpetu-wakan oko ehake kin he toked eciyapi he? Wokakije Anpetu-wakan Oko, qa nakun Anpetuwakan Oko Wakan kin.

\section{WOONSPE XXXIX.}

1. Anpetu wan ed Jesus canicipawega akand okatanpi qon he toked eciyapi he? Good Friday.

2. Saturday wan ed wicarapi ed wanke ciqon he toked caxtonpi he? Easter itokab Rtayetu kin.

3. Tohonwind wicate cir etanhan kini he? Easter Anpetu kin ed.

4. Anpetu wan ed wankand marpiyata iyaye ciqon he toked caxtonpi he? Ascension-Anpetu.

5. He tohantu he? Easter iyohakab anpetu wikcemnatopa. 
6. Tohonwind Jesus Woniya Wakan kin waonspewicakiye cin ed hiyuya he? Wankand iyaye cin iyohakab anpetu wikcemna qehan.

7. Anpetu kin he toked caxtonpi he? Anpetu-wakan-ska.

8. Hetanhan anpetu ixakdogan kin he toked caxtonpi he? Yamni Taanpetu-wakan kin.

\section{WOONSPE XL.}

1. Saint Mark unkiksuyapi kta e tukte anpetu kae ahounpapi he? April 25th.

2. Saint Mark toked eciyapi he? Wotanin-Waxte Owa kin.

3. Mark eciyapi kin he tuwe he? Juda oyate Levi tiyoxpaye kin etanhan.

4. Toketu rcin cajeyatapi he? John Mark.

s, Tuwa he yuhomni qa Christian hecaya he? Peter Wahoxiye wakan kin.

6. Ix Peter taku ecakicon he? Iekiciska.

7. Mark Wotanin-waxte kin tuwa owicakiyaka he? Egypt ed ikcewicaxa yukanpi kin.

8. Toked ta he? Ix eya Martyr heca.

\section{WOONSPE XLI.}

1. Anpetu wanji tukte ed Saint Philip qa Saint James wicunkiksuyapi he? May 1st.

2. Philip eciyapi kin he tuwe he? Wahoxire Wakan.

3. He Christ ed tuwa ai he? Nathanael.

4. Philip toked ta he? Canicipawega ed olkatanpi.

5. Saint James eciyapi kin he tuwe he? He Alpheus cinhintku. 


\section{7}

6. Pharisee kin taku ecakiconpi he? Tipi-Wakan oinkpa kin eciyatanhan kar'odiyeyapi.

7. On ta he? Hiya; inyan on ktepi.

8. Saint James unma kin he toked eciyapi he? Saint James Wahoxiye Wakan kin.

9. Tuwa he cinhintku he? Zebedee.

\section{WOONSPE XLII.}

1. Saint Barnabas taanpetu kin tohantu he? June 11th.

2. Caje tokaheya kin tukte e he? Joses.

3. Barnabas eyapi kin he taku kapi he? Wokicanpte cinhintku kin.

4. Toka e heya caxtonpi he? Warpanicapi kin on tamaga wiyopekiye cin heon.

5. Hehand ake wokiksuye anpetu wanji kin he tukte he? Saint John Baptisma wicaqu taanpetu kin.

6. He tohantu he? June 24th.

7. St. John tuwa ateya he? Zacharias.

8. Saint John Baptisma wicaqu qon he tuwe he? He Christ canku pikiciciya.

9. Taku cajeyad wahokonwicakiya he? Woiyopeiciye. 10. Toked ta he? Owicakaxke tipi wan ed pa kaksapi.

\section{WOONSPE XLIIJ,}

1. Saint Peter taanpetu kin tahantu he? June 29.

2. Saint Peter toked wartani he? Itaucan tawa kin sdodye xni konza.

3. On icantexica he? Han; on nina iyopeiciya.

4. He toked ta he? Canicipawega ed okatanpi.

5. Saint James taanpetu kin tohantu he? July 25. 


\section{8}

6. Jt. James Wahoxiye Wakan kin he tuwe he? Zebedee qa Salome cinhintkupi.

7. He tokod ta he? Acts xii:1. Herod miwakan wan on kte.

8. Saint Bartholomew taanpetu kin tohantu he? August 24 .

9. Caje tokaheya kin tukte e he? Nathanael.

10. He toked ta he? Niyake ha yuzapi, qa hehand canicipawega ed okatanpi.

\section{WOONSPE XLIV.}

1. Saint Matthew taanpetu kin tohantu he? September 21 st.

2. Saint Matthew eciyapi kin he tuwe he? Wahoxiye Wakan qa Wotanin-waxte owa kin heca.

3. Taku wicor'an kae etanhan Christ he kico he? Wamnayanpi.

4. Jesus taku eciya he? "Miyakna u wo."

5. Tukte ekta ktepi he? Ethiopia ed.

6. Anpetu wan ed Saint Michael qa Oknikde Wakan owasin wicunkiksuyapi kin he tohantu he? September 29.

7. Saint Michael taku econ he? Rev. xii: 7,9. Marpiya kin etanhan Satan tokan iyeya.

8. Oknikde Wakan eyapi kin he taku hc? Wakantanka he waeconxi eca toie anagoptan kin heca.

\section{WOONSPE XLV.}

1. Saint Luke taanpetu kin tohantu he? October 18.

2. Saint Luke eciyapi kin he tuwe he? Wotanin Waxte owa kin heca.

3. He taku wicaxa he? Pejuta-wicaxa. 


\section{9}

4. Taku on ocaxtonka he? Takuxnixni ite owa wayupika on.

5. Nakun taku on icajeyatapi s'a he? "Waonspewicakiya wikcemna xakowinpi" wanji ee kin he.

6. He tuwa kici ounyesa he? Saint Paul.

7. Tohonwind? Paul kaxka yanke ciqon he ehan.

8. Luke Wotanin-Waxte owa kin isakab nakun taku owa he? "Wahoxiye Wakan Or'anyanpi qon" hena.

9. Toked ta he? "Wikdi can wan ed otkeyapi" keyapi.

\section{WOONSPिE XLVI.}

1. October 28th en Wokiksuye anpetu kin he toked eciyapi he? Saint Simon qa Saint Jude taanpetupi kin.

2. Simon eciyapi kin he tuwe he? Simon Zelotes, Wahoxiye Wakan kin.

3. Zelotes eyapi kin he taku kapi he? Natonke xni rcin xkan kin, qaix aiciciye rcin xkan kin.

4. Tokae akta rcin caje kin de qupi he? Wanji ix eya Simon eciyapi kin heon.

5. Unman kin he tuwe he? Simon Peter.

6. Zelotes on taku sdodunyanpi he? Wahoxiye Wakanpi kin etanhan hecena rein kitanecinyan sdodunyanpi.

7. Saint Jude eciyapi kin he tuwe he? He Cleophas cinhintku.

8. Toked ta he? Kakixyapi on ta.

9. Jude taku owa he? Jude owancaya wowapi wicakicage cin.

10. Wokiksuye anpetu ehake rce cin he tukte e he? Wakanpi owasin taanpetupi kin.

11. He tohantu he? Novemuer 1st.

12. He taku ikiksuyeunyanpi he? Wakanpi odakodkiciyapi kin.

13. David tuwa owotanna kin he taku ecakiconpi kta 
keya he? "Wicaxa owotanna kin he owihanke wanin kiksuyapi kta."

\section{WOAHOPE WIKCEMNA KIN.}

1. Wakantanka woahope etanhan wicaqu he? Han.

2. Tuwa hena qu he? Moses.

3. Tuwa hena yuhapi kta e hecon he? Israel oyate kin.

4. Qa nakun tuwa? Unkiyepi.

5. Tukte ed hena wicaqu he? Sinai Paha kin akand.

6. Sinai Paha kin he tuktetu he? Arabia makoce kin ed.

7. Taku akand owapi he? Inyan bdaska nonb.

8. Wowapi wakan kin tukte ed woahope kin nena iyeunyanpi he? Kdinapapi, Wicowoyake 20.

9. Waohope kin tonakeca he? Wikcemna.

10. Hena owasin ahopenixipi he? Han.

11. Woahope tokaheya kin he tukte e he? Mitokab taku wakan tokeca duha kte xni.

12. Icinonpa kin he tukte e he? Wakagapi wanjina itokab cankpexka makakde inayajin kte xni.

13. Iciyamni kin he tukte $\Theta$ he? Wakantanka eaje kin he ituya ehin kte xni.

14. Icitopa kin he tukte he he? Anpetu Okirpapi kin wakanyan duha kte cin he kiksuya yo.

15. Icizaptan kin be tukte e he? Niyate qa nihun wicakduonihan yo.

16. Icixakpe kin he tukte e he? Tidwicaktepi ecanon kte xni.

17. Icixakowin kin he tukte e he? Wicoie qa wicoran xice cin hena etanhan dehanyag ikduha yo.

18. Icixakdogan kin he tukte e he? Wanirpanica qa onnixika exa takuna manon xni yo.

19. Inapcinwanka kin he tukte $\mathrm{e}$ he! Owewakankan xni qa waxtedake xni.

20. Iwikcemna kin he tukte e he? Nikiyesa-ti kin tawa kin cantiheye xni yo. 


\section{1}

\section{IYOKIHEYAPI.}

I. Wicaxa tokaheya qon he tuwe he? Adam.

2. Winyan tokaheya qon he is tuwe he? Eve.

3. Tokaheya tidwicalste qon he tuwe he? Cain.

4. Wicaxa wan iyotan tehan ni qon he tuwe he? Methuselah.

5. Wicaxa wan iyotan wax'ake ciqon he tuwe he? Samson.

6. Wicaxa wan iyotan onxiicida qon he tuwe he? Moses.

7. Wicaxa wan iyotan ksape ciqon he tuwe he? Solomon.

8. Wicaxa wan iyotan wacin tanka qon he tuwe he? Job.

9. Mnaja tipi wan mahed irpeyapi qon he tuwe he? Daniel.

10. He tuwe e kangi woqupi he? Elijah.

11. Hunkawanjitku wiyopekiyapi qon he tuwe he? Joseph.

12. Mini cin qa on imnija wan ape ciqon he tuwe he? Moseș.

13. Tanzaniyake Paradise ekta iyayapi qon hena tuwepi he? Enoch qa Elijah.

14. Wakantanka wicaxa wan iyokipi qon he tuwe he? David.

15. He tuwe e anpetu wi kin owanji inajinxi he? Joshua.

16. He tuwe e watokapa wiyopekiye he? Esau.

17. Tuwa he wiyopekiya he? Jacob.

18. Tuwa witawàta tanka wan kaga he? Noah.

19. Wiwazica cinhintku niye ciqon he tuwe he? Elijah waayata kin.

20. Hogan tanka wan napce ciqon he tuwe he? Jonah.

21. Miniskuya-paha icage ciqon he tuwe he? Lot tawicu kin.

22. Wicaxayatapi xica wan oceti ideyahan ed oiyorpe- 


\section{2}

wicaye ciqon hena tuwepi he? Shadrack, Meshach qa Abednego.

23. Hunku Itancan kin ed oqu qon he tuwe he? Samuel.

24. He tuwe e toni kin he Wakantanka waniyetu akezaptan akiciyawa he? Hezekiah wicaxayatapi waxte kin.

25. David inyan iyurmun ed inyan oknanke ca on tuwa kte he? Goliah.

26. Wakantanka tuwa econxi qa on Miniwanca Xa mini kin yukinukan he? Moses.

27. Tuwa Jordan wakpa kin taxina on ape ca mini kin yukinukan he? Elijah.

28. Tuwa "Ceyaya waayates'a" eya cajeyatapi he? Jeremiah.

29. Tatanka iyeced peji yute ciqon he tuwe he? Nebuchadnezzar.

30. Psalm iyuha kinind owa qon he tuwe he? David.

31. Wakantanka oyate wan karnige ciqon he tukte e he? Juda oyate kin.

32. Ikcewicaxa rca ekna Wahoxiye wakan un qon he tuwe he? Saint Paul.

33. Canicipawega wan akand kud itoheya okatanpi qon he tuwe he? Saint Peter.

34. Wikdi piryahan ed oiyorpeyapi qon he tuwe he? Saint John.

35. Hokxicopa ehantanhan Wowapi Wakan kin slodye ciqon he tuwe he? Timothy.

36. Warpanicapi kin hayake wicakicage ciqon he tuwe. he? Dorcas. Oran. ix.

37. Woowewakankan on ta iyaye ciqon he tuwe he? Ananias.

37. Qa nakun tuwa? Sapphira.

39. Christian Martyr tokaheya qon he tuwe he? Saint Stephen. 
Microtormed by

Preservation

Services

mim $\frac{6532.01}{275.95}$. 


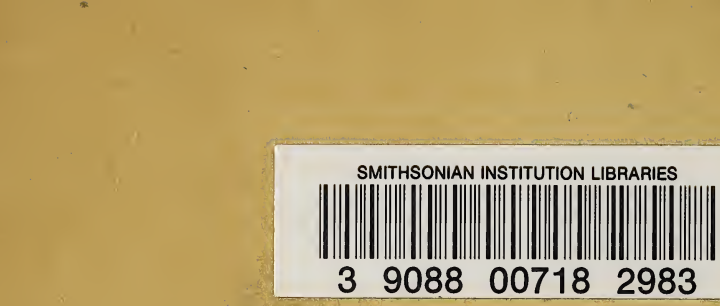

